\title{
COOL, \\ LCG Conditions Database for the LHC Experiments: Development and Deployment Status
}

\author{
Andrea Valassi, Romain Basset, Marco Clemencic, Gianni Pucciani, Sven A. Schmidt, Martin Wache
}

\begin{abstract}
The Large Hadron Collider (LHC), the world's largest and highest-energy particle accelerator, designed to collide opposing beams of protons or lead ions, started its operations in September 2008 at the European Organization for Nuclear Research (CERN) in Geneva, Switzerland. To process and analyze the huge amounts of data generated by the four experiments installed at different collision points along the LHC ring, a large distributed computing infrastructure has been set up, the LHC Computing Grid (LCG). The bulk of this data, referred to as 'event data', will record the signals left in the subdetectors of the four LHC experiments by the passage of the particles generated in the collision of the LHC beams. A different set of data, referred to as 'conditions data' and needed for the analysis of event data, will record the experimental conditions at the time the event data were collected, such as the measured temperatures or the calculated calibration factors for the several sub-detectors of each LHC experiment.

The COOL project provides common software components and tools for the handling of the conditions data of the LHC experiments. It is part of the LCG Persistency Framework (PF), a broader project set up within the context of the LCG Application Area (AA) to devise common persistency solutions for the LHC experiments. COOL software development is the result of the collaboration between the CERN IT Department and ATLAS and $L H C b$, the two experiments that have chosen it as the basis of their conditions database infrastructure. COOL supports conditions data persistency using several relational technologies (Oracle, MySQL, SQLite and FroNTier), based on the CORAL Common Relational Abstraction Layer. For both experiments, Oracle is the backend used for the deployment of COOL database services at Tier0 and Tier1 sites of the LHC Computing Grid. While the development of new software functionalities is being frozen as LHC operations are ramping up, the main focus for the project in $\mathbf{2 0 0 8}$ has shifted to performance optimization for data insertion and retrieval and to the deployment and test of Oracle database services for COOL. In this presentation, we will review the status and plans of both software development and COOL database service deployment at the time of the NSS conference, a few weeks after the start-up of the LHC.
\end{abstract}

Manuscript received November 28, 2008

A. Valassi (e-mail: Andrea.Valassi@cern.ch) and R. Basset (e-mail: Romain.Basset@cern.ch) are with the IT-DM Group at CERN, Geneva, Switzerland.

G. Pucciani (e-mail: Gianni.Pucciani@cern.ch) was with the IT-DM Group and is now with the IT-GD Group at CERN.

M. Clemencic (e-mail: Marco.Clemencic@cern.ch) is with the PH-LBC Group at CERN. He is a member of the LHCb collaboration.

S. A. Schmidt (e-mail: sas@abstracture.de) and M. Wache (e-mail: wache@abstracture.de) are with Mainz University, Germany. They are members of the ATLAS collaboration.

\section{INTRODUCTION}

$T^{H E}$ LCG Conditions Database project [1] was launched in July 2003 with the goal of implementing a common persistency solution for the storage and management of the conditions data of the LHC experiments at CERN. The project was initially active [2] in collecting the requirements of the experiments in this area and in reviewing the limitations of several pre-existing software packages. Eventually, two LHC experiments (ATLAS and $\mathrm{LHCb}$ ) decided to join forces for developing a new software package that would better address their needs. Additional resources for software development were provided by the CERN IT Department, which also took on the role of the overall project coordination.

As requested by the experiments, the new software package, COOL (Conditions Objects for LCG) [3], provides support for several relational database technologies, including Oracle and MySQL servers, the SQLite file-based SQL database engine and the FroNTier web-based database access system. Taking into account the limited resources available for its development, the design of COOL was driven from the start by the need to avoid all duplication of effort, both with respect to other ongoing LCG developments and internally amongst the different use cases and persistent technologies supported. Of particular relevance in this context were the choices to base the relational implementation of COOL on the CORAL Common Relational Abstraction Layer (also delivered by another LCG common project) and to adopt exactly the same relational schema for all back-ends.

The project provides a set of $\mathrm{C}++$ libraries implementing abstract interfaces that describe the functionalities required by the users, as well as Python wrappers of the same interfaces and tools for COOL data management. The core functionality of the COOL software consists in managing the time variation and, where relevant, the versioning of conditions data. While the main use case is rather simple - users need to store and later retrieve a set of conditions data objects (or "IOVs", for "intervals of validity") valid in a given time range and for a given tagged version - COOL provides several hooks to customize the storage and retrieval of conditions according to the data model most appropriate to each user, e.g. using data payload of different data types or conditions metadata involving several channels or several versioning mechanisms.

The initial phase of development [3], from the first production release COOL 1.0.0 in February 2005 to about 
mid-2006, delivered the implementation of the basic functionalities required by the experiments. The following phase of development [4], leading to the COOL 2.0.0 release in January 2007 and later the COOL 2.2.0 release in July 2007, focused on the consolidation of the COOL software using an improved $\mathrm{C}++$ API and a more flexible relational schema. The main motivations for these backwardincompatible API and schema changes were the need to support a few new functional requirements, the port to 64-bit platforms and the improvement of client-side performance.

Since the beginning of 2007, with the approach of the LHC start-up date and in parallel to the progress in the deployment of COOL Oracle services at Tier0 and Tier1 LCG sites, the focus of development has progressively shifted to the serverside query optimization for data retrieval from, and insertion into, Oracle databases. During a first round of performance optimization studies in 2006 and 2007 [5], several use cases were individually analyzed and optimized. The highest priority was given to those use cases where performance problems had been pointed out by the large scale tests performed by the experiments using their distributed database services already deployed at Tier0 and Tier1 sites [6].

In this paper, the recent developments in COOL in late 2007 and 2008 and the plans for 2009 will be reviewed, with particular emphasis on the description of performance tests and optimizations. The updated status and plans for the deployment of COOL services at Tier0 and Tier1 sites will also be presented.

\section{COOL DEPLOYMENT - STATUS AND OUTLOOK}

The LCG 3D (Distributed Deployment of Databases) project [7] is a joint activity between LHC experiments and LCG tier sites to coordinate the setup of relational database services and data transfer facilities as part of the LCG infrastructure. Its goal is to provide a consistent way of accessing database services at CERN Tier0 and collaborating LCG tier sites.

The deployment of relational database services hosting the COOL data of the LHC experiments on the LCG tier sites follows the general architecture model that was proposed and implemented in the context of the 3D project. This model, shown on Fig. 1, foresees the complementary use of a variety of relational database technologies. The Oracle 10g RAC (Real Application Cluster) technology, in particular, was chosen to set up relational database services at CERN Tier0 and at all collaborating Tier1 sites. The model generally foresees separate Oracle clusters at CERN Tier0 for the different LHC experiments, with two different clusters set up for each experiment to host the "online" data relevant to datataking, and the "offline" data relevant to the later processing and analysis steps.

Data replication, between the online and offline clusters at Tier0 as well as from Tier0 to all Tier1 sites, is ensured in this model by the Oracle Streams technology. Data changes are captured at the source database and propagated to the target database for replication, where they are then applied so that the target database contents are synchronized with those of the source database.

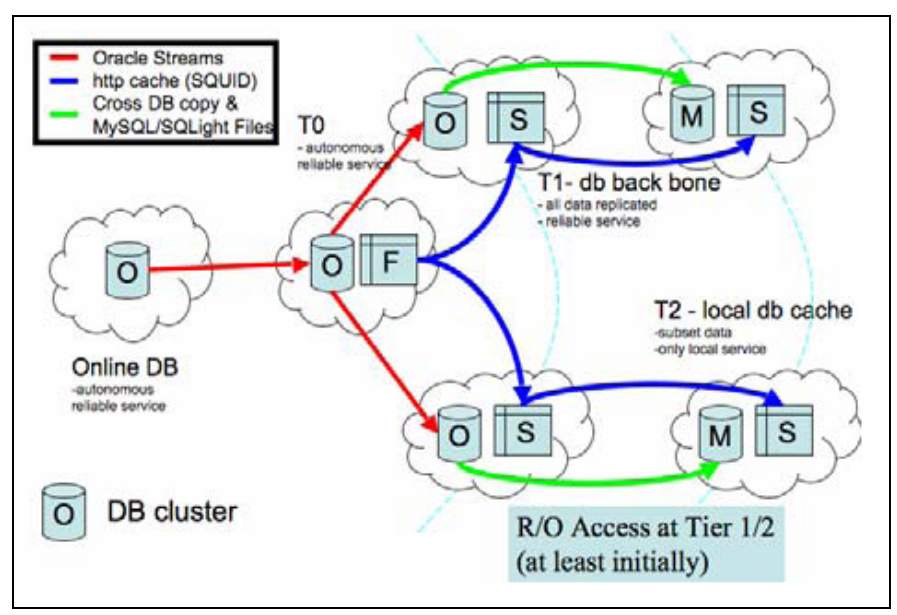

Fig. 1 - LCG 3D architecture model for the setup of relational database services and data transfer facilities at LCG tier sites. The model foresees the complementary use of a variety of relational database technologies, including Oracle (O), MySQL (M), SQLite (S) and FroNTier (F).

The complex infrastructure necessary to implement this model has been gradually put in place over the last couple of years [8]. At the time of writing, the COOL deployment setup is finally complete for both ATLAS and LHCb, at Tier0 (both online and offline) and at all relevant Tierl sites (six for $\mathrm{LHCb}$, ten for ATLAS). As it will be described in chapter III, these setups are now being extensively used and tested by the experiments. The data volumes are much larger in ATLAS than in LHCb: this can be appreciated qualitatively in Fig. 2, showing the data rates for the Oracle Streams LCRs (Logical Change Records) propagated from Tier0 to the various Tier1 sites during the week preceding the NSS 2008 Conference.

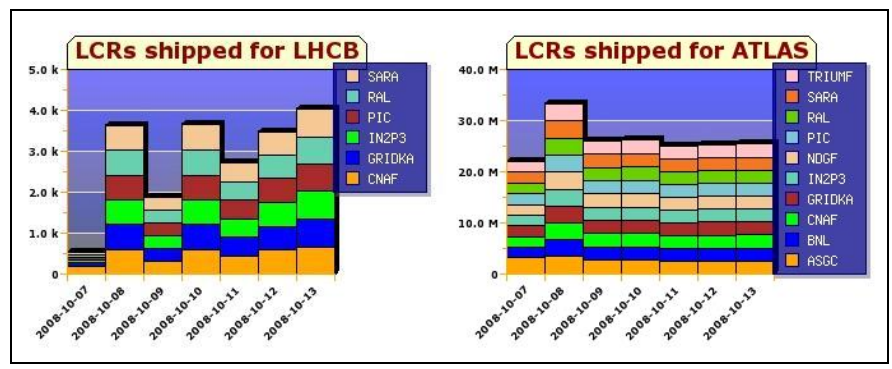

Fig. 2 - Data rates for "Oracle Streams" replication from CERN Tier0 to the different LCG Tier1 sites (six for LHCb, ten for ATLAS) during the week preceding the NSS 2008 conference. Data rates are expressed in Oracle Streams LCRs (Logical Change Records) per second.

\section{A. COOL deployment model with a CORAL Server}

In the deployment model described above, currently all COOL user applications open direct connections to the relevant Oracle database servers. From the software point of view, user applications invoke methods of the COOL API, implemented inside COOL by dispatching all relational database operations to the CORAL API. If the COOL URL 
specified by the user at connection time points to an Oracle server, CORAL internally loads the plugin responsible for accessing Oracle databases, which is implemented using the Oracle OCI client library. A direct connection from the user application to the database server is thus established via OCI. This is shown schematically on the left side of Fig. 3.

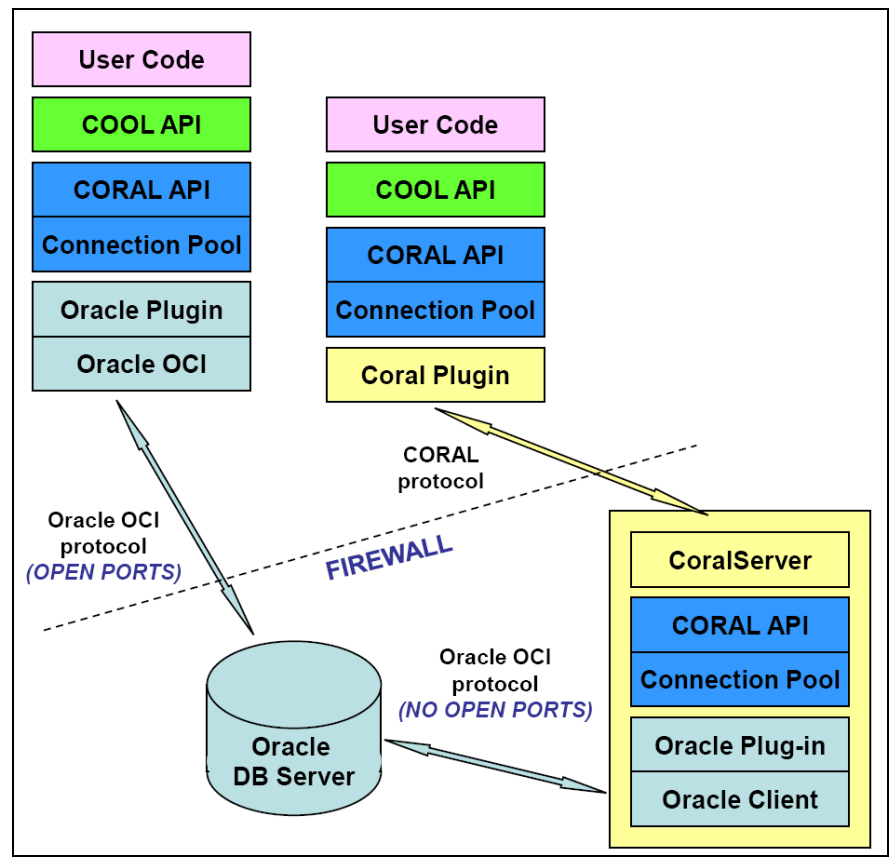

Fig. 3 - Connection of a COOL client program to an Oracle database in a deployment model without (left) and with (right) a CORAL Server.

Several limitations of this connectivity model are becoming apparent with the growing use of the deployed servers by COOL and other applications. To start with, the only authentication mechanism currently supported for the experiment jobs consists in providing user names and passwords at connection time. This poses several security risks, including password vulnerability and the need to expose Oracle server ports on the public network for remote users. Another limitation of the model is that different client jobs must establish separate physical connections to the Oracle server, even when they all access the same schema using the same credentials. This may lead to a very inefficient use of server resources, resulting in performance bottlenecks when connection rates are high.

Improvements on these and other issues may come in the future from a new connectivity model that has recently been proposed. The basic idea is that a middle-tier server should be introduced between client applications and the database servers. CORAL-based clients, including COOL applications, would connect to this "CORAL server" through a new dedicated CORAL plugin and a new custom protocol, while the CORAL server, itself a CORAL application, would connect to the Oracle database server inside the site firewall. This is shown schematically on the right side of Fig. 3. Development for all the relevant new components has already started: the status and outlook are discussed in detail in another contribution to this conference [9].

From the point of view of COOL, it is expected that only minor changes in the software would be needed, to support a new URL type. The advantages of the new connectivity model would all apply to COOL clients. To start with, authentication via proxy certificates on the CORAL server would remove the need for COOL users to provide explicit user names and passwords. In addition, several connections from different client hosts to the same Oracle database server could be multiplexed through the CORAL server, resulting (in the ideal case) in a single physical connection from the CORAL server to the Oracle server. As it will be discussed later in section III.B, it has been reported that reducing the number of Oracle sessions opened by ATLAS COOL applications is desirable to decrease the load on the database servers: this is just one specific example where the multiplexing offered by the CORAL server could be very useful.

\section{COOL DEVELOPMENT - STATUS AND OUTLOOK}

Four years after the start of its development, the COOL software is by now mature in terms of the spectrum of functionalities it provides, the stability of its C++ API and relational schema and the reliability of its code base.

Reliability, in particular, has been consistently pursued through the lifetime of the project by the emphasis given to functional testing. From the start, a comprehensive test suite was developed in parallel to the implementation code, allowing the same functional tests to be executed repeatedly, against each of the supported relational back-ends. Since February 2007, these tests are automatically run every night using the latest unreleased version of the code, in the wider context of the nightly build and test system set up for all LCG AA common software projects.

While the development of new software functionalities is almost frozen as the experiments require stable software packages at the time of the LHC start-up, some consolidations of COOL triggered by changes in external software, the need to support new platforms and compilers, as well as a few minor feature extensions, are still ongoing. The main emphasis of COOL development activities, however, has steadily moved to performance tests and optimizations: these activities have been the main focus of the project in 2008 and are expected to be also in 2009 .

\section{A. Software consolidation and functional enhancements}

\section{1) Removal of COOL dependencies on SEAL}

One of the most important consolidations of the COOL software completed in 2008 was the removal of all its dependencies on the SEAL package. This change was triggered by the AA decision to discontinue support for the SEAL project, which was formerly providing the $\mathrm{C}++$ core software libraries and services for all AA common packages. Most components of SEAL were moved to other AA common projects (in particular, ROOT) or to the experiment-specific 
frameworks, others were replaced by similar ones providing the same functionalities in BOOST, a few were dropped.

As far as COOL is concerned, the transition out of SEAL had already started in previous years. As early as January 2006, the PyCool component (providing Python bindings for the COOL $\mathrm{C}++\mathrm{API}$ ) had been re-implemented using the Reflex (C++ reflection) component from ROOT instead of that from SEAL. In the 2.0.0 release in January 2007, in view of the phasing out of this package, the SEAL classes and type definitions used to describe time and 64-bit integers in the COOL $\mathrm{C}++$ API had been replaced by equivalent ones not based on SEAL. The API change had also involved the replacement of the CORAL AttributeList by a custom COOL Record class.

What still needed to be done was the removal of the dependency of COOL on the SEAL component model and dynamic plugin loading infrastructure. This step was more complex than the previous ones because it depended on the simultaneous completion of the same task in CORAL, as the SEAL component model acted as the glue connecting COOL and CORAL together in a single software framework and allowing end-user applications to issue calls to both packages. This was eventually achieved in COOL 2.5.0 and CORAL 2.0.0, released together in June 2008 as part of the LCG_55 configuration of the LCG AA software stack. In CORAL, the SEAL component model and plugin loader were internally replaced by custom ones, while the ConnectionService class was exposed in the public API as the single point of entry into the CORAL framework. In COOL, the need for a plugin loader was removed by turning the RelationalCool shared library from a loadable plugin into a link-time dependency of all COOL-based applications. The COOL Application class was also modified to provide mechanisms to bootstrap COOL from an existing CORAL ConnectionService, and/or retrieve an instance of the latter from COOL even when this is bootstrapped in standalone mode.

\section{2) Port to new platforms}

In parallel to the upgrade to new versions of external software, another routine maintenance task for COOL is its port to new platforms and compilers. In the LCG AA policy, all projects of the common software stack are released together several times per year, using pre-defined versions of all needed external packages and providing supported builds for an agreed set of platforms and compilers. The detailed requirements for each new $\mathrm{LCG}$ AA software release are routinely discussed and agreed by the architects of the four LHC experiments and of the common software projects at biweekly "Architects Forum" meetings.

During most of 2007, all LCG common software, including COOL, was supported on several flavors of two platforms: Scientific Linux CERN (32-bit SLC3, using the gec 3.2.3 compiler, as well as 32-bit and 64-bit SLC4, using gcc 3.4.6) and Microsoft Windows (32-bit, using the Visual $\mathrm{C}++7.1$ compiler). In the COOL 2.2.1 release in October 2007, SLC3 was dropped, while support for a third platform was added: Mac OSX 10.4 (32-bit, on Intel processors only, using the gec
4.0.1 compiler). This was eventually replaced by OSX 10.5 in COOL 2.5.0 (June 2008), while support for Oracle on this platform was finally added in COOL 2.6.0 (November 2008).

At the time of writing, work is in progress on completing COOL support for two new compilers, gec 4.3.2 on SLC and Visual C++ 9.0 (Visual Studio Express 2008) on Windows, as well as on the port to the SLC5 Linux distribution. Support for these new platforms and compilers will most likely be added in a new LCG release before the end of 2008.

\section{3) Functional enhancements}

As discussed above, even if the spectrum of functionalities provided by the COOL software is by now wide and solid enough to satisfy the vast majority of use cases, a few functional enhancements are still being implemented as requested by the LHC experiments.

Several such features have been added in recent releases in the area of versioning and tagging. As a reminder, in the COOL terminology a "tag" is a collection of IOVs such that at any given point in time only one specific version of each data item is present in the tag (for instance, a calibration tag may include for each IOV the calibration for that time range as computed using a well defined algorithm). Examples of newly added features include methods for "locking" tags so that they cannot be modified, except at most for the addition of fresh IOVs ("partial locking"), or the possibility of creating a new tag from an existing one, so that the two can be modified independently ("tag cloning"). Partial tag locking and tag cloning have both been completed in the most recent release, COOL 2.6.0 in November 2008.

The COOL 2.6.0 release also includes another important new feature of a very different type. This new functionality, known as "payload queries", allows users to search for the IOVs during which the conditions data "payload" of the given data item satisfies some user-defined selection criteria: for instance, users may now query the COOL conditions database to identify the time ranges during which a temperature was higher than a given threshold. This query mechanism is the inverse of the most common use case for which COOL was originally designed, the lookup of the data payload valid during a given time range (most often, with the goal of using that data payload in the analysis of the event data collected during that time range). In specific cases, such as the selection of a range of payload values, COOL allows payload queries to be executed on the database server by translating the selection criteria into SQL fragments.

Last but not least, a few new deployment-oriented features are currently under development. One such functionality is the support for the CORAL server backend, as previously mentioned in section II.A. Other important improvements, relevant to transaction and session management, will be mentioned later in section III.B. Finally, a few enhancements to the COOL relational schema are being prepared, such as the option to store IOV metadata and the corresponding conditions data payload in two separate sets of tables, so that several IOVs can refer to the same data payload, and the option to use the same sets of tables for different data items as long as their payload specification (the set of required payload columns) is exactly the same. All of these enhancements may 
be added in future COOL releases, although no definite plans exist yet in this direction.

\section{B. Performance optimizations}

As previously mentioned, performance tests and optimizations have been the main focus of the COOL project throughout 2008, and are expected to be also in 2009. Largely speaking, three types of activities have been going on in this area. To start with, proactive performance tests and optimizations using small simulated data sets have been continued in a very systematic way, to devise the best SQL query strategies for all of the most important use cases for data insertion and retrieval. Another area of activity has involved scalability tests for data insertion and retrieval using very large samples of simulated data, emulating the table sizes resulting from several years of data taking in an LHC experiment. Finally, support has been provided to the experiments to solve any high-priority performance issues identified during the tests they performed using their final deployment setup for data taking.

Since Oracle is the backend chosen for the deployment of COOL databases at Tier0 and Tier1 sites, as previously discussed in chapter II, performance tests and optimization have mainly concentrated on this technology. All of the results that will be presented in this section refer to Oracle, unless otherwise stated. At the same time, some minor optimizations for the other backends, most notably MySQL, have also been added to COOL. In particular, this has involved using different SQL queries in the few cases when the optimal SQL strategy for Oracle produced suboptimal results on the other backends; with the exception of these few cases, the same SQL queries were otherwise used on all backends.

\section{1) Basic SQL tests and optimizations}

Proactive performance tests and SQL optimizations based on small simulated data sets have been a key tool in the COOL project for several years by now.

The basic idea is that the retrieval of IOVs valid in a given time range should be optimized so that the response time for the corresponding queries is not only as low as possible, but it is also the same independently of whether the IOVs being queried correspond to very old or very recent time ranges. From the point of view of the implementation of COOL using relational database technologies, this generally corresponds to saying that SQL queries on the tables containing those IOVs should be based on indexes rather than proceed via the full scans of those tables.

Simple plots of the query response time for the IOVs around validity time $T$, as a function of validity time $T$, are shown in Fig. 4 for the specific use case of "multi-version retrieval from a tag". Validity time $\mathrm{T}$ is measured in arbitrary units, irrelevant for the sake of this discussion: it will be enough to note that the tables used for these tests are very small (on LHC conditions data standards), as they contain a few hundred thousand rows at most.
Several curves are shown in Fig. 4 for different COOL releases and under several scenarios in each release, as it will be discussed later on. Without going into the details yet, note that the red curve displaying the highest slope corresponds to the suboptimal SQL query used in release COOL 2.3.0, resulting in a full table scan: in simple terms, querying data at the beginning of a table (low values of T) is faster than querying data at the end of a table (high values of $\mathrm{T}$ ) if the strategy involves a full scan of the table ordered by increasing values of $T$. The light blue curve that exhibits no increase in response time as a function of $\mathrm{T}$, conversely, corresponds to the new optimal SQL query defined in release 2.3.1, resulting in indexed data access to the IOV table: query times over a binary index only increase logarithmically, meaning that they are essentially constant for most practical purposes.

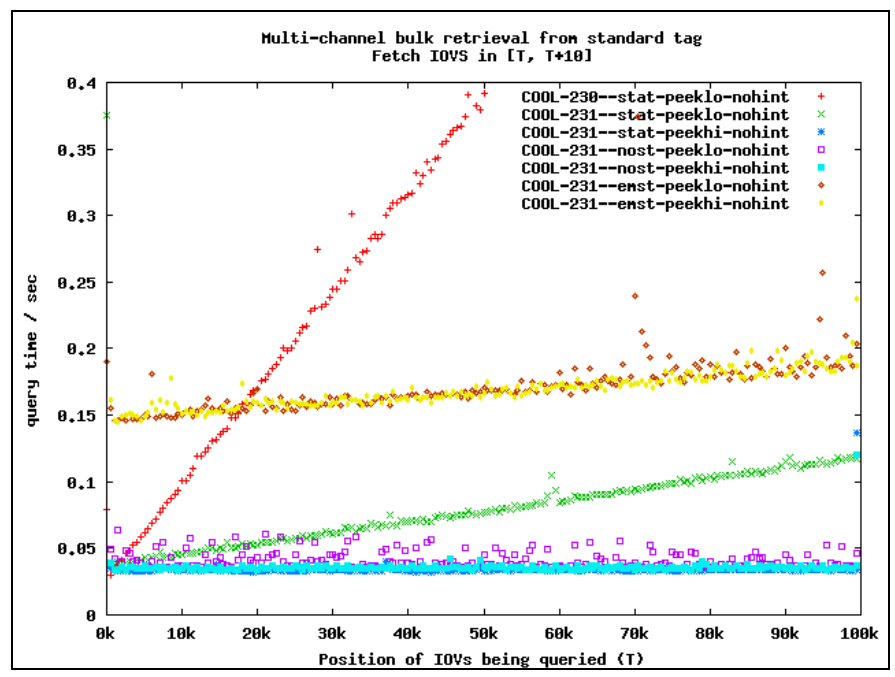

Fig. 4 - Query response times (seconds) for the retrieval of all IOVs in the time range $[\mathrm{T}, \mathrm{T}+10]$, as a function of $\mathrm{T}$ (arbitrary units), in the specific use case of "multi-version retrieval from a tag". One curve is given for release COOL 2.3.0 and the other six for release 2.3.1, under several scenarios of "statistics gathering" and of "bind variable peeking", as explained in the text. Oracle Optimizer "hints" are disabled for the curves plotted for 2.3.1.

Many SQL optimizations of the kind described above, turning a steeply increasing response time curve into a flat one, had already been achieved for various use cases in several COOL versions released in 2006 and 2007 [5]. What changed in 2008 is that two important new improvements were completed. To start with, a better understanding of the possible causes of performance instabilities for a given SQL query (as described in detail in the next paragraphs) led to the decision to add Oracle Optimizer "hints" to all COOL queries. In addition, the $\mathrm{C}++$ implementation code was rewritten to make sure that few methods describing SQL queries with well defined performances are called for a large variety of different use cases.

One of the first clear indications of performance instabilities in the SQL queries issued by older COOL releases came from the ATLAS distributed database tests in 2007. It was reported in that occasion that very different performances were observed at two ATLAS Tier1 sites, in Bologna and Lyon. 
This was eventually understood as being caused by differences in the reliability of the Oracle Optimizer "statistics" for the database servers installed at the two sites.

The Oracle Optimizer is the internal component of the Oracle server software that is responsible for determining the "execution plan" according to which a given SQL query should be executed, for instance, using one combination of indexes or another. Several factors are taken into account in this algorithmic decision, one of them being the distribution of values in the table columns that are relevant to the selection criteria in the query. If the internal histograms (the so-called "statistics" previously mentioned) describing these distributions are not frequently updated as more data are added to the database, the execution plan chosen for the query on a set of tables may not be the optimal one to be applied given the current contents of those tables. This is exactly what had happened in one of the two ATLAS Tierl sites above.

A second important factor in the determination of the execution plan for an SQL query may be the value of the "bind variables" associated to the query selection criteria. In the example of the retrieval of IOVs valid around time T, different execution plans may be optimal depending on the values of $\mathrm{T}$. A typical example is that for $\mathrm{T}=0$, where the IOVs to be retrieved are the first ones in the respective tables, a full table scan may be just a bit more efficient than indexed access. The problem in this case comes from a feature of Oracle known as "bind variable peeking" (in the Oracle server version $10 \mathrm{~g}$ used by most COOL deployments): if a query is executed once for a given set of bind variables, the same execution plan is normally kept also for the next execution using a different set of bind variables. This feature is generally a good optimization (as it is expensive to compute an execution plan), but in this case it is a nuisance because the execution plan optimal for low values of $\mathrm{T}$ may be suboptimal for high values of $\mathrm{T}$.

Having identified statistics reliability and bind variable peeking as the two main factors for SQL query stability, their effect is now systematically studied in all COOL performance tests. The six curves shown in Fig. 4 for the COOL 2.3.1 release, in fact, correspond to all combinations of two scenarios for bind variable peeking (low or high values of T) times three scenarios for statistics (reliable statistics, unreliable statistics computed on empty tables, no statistics). In Fig. 4 it is clearly shown that several such combinations lead to poor performance for some or all values of $\mathrm{T}$.

As it was briefly mentioned, the strategy that was developed to remove the execution plan instabilities due to statistics reliability or bind variable peeking consists in systematically adding Oracle Optimizer "hints" to all SQL queries in COOL. The practical strategy that was used to determine the hints most appropriate to each query is described in detail in another presentation [10]. In short, this was based on the analysis of the BEGIN_OUTLINE_DATA sections in the "event 10053" Oracle trace files for queries executed, in the absence of Oracle hints, in the six scenarios previously described (i.e. for the scenarios corresponding to the six curves in Fig. 4). These sections of the trace files describe the execution plans used for each query, using a syntax that can be used to define hints that would result in the same execution plan. By looking at those scenarios that result in good performance even in the absence of hints, this iterative procedure eventually allowed the definition of hints resulting in the same execution plan and the same good performance also in the other scenarios. The results are shown in Fig. 5: when hints are applied (which is the default in COOL 2.3.1), query performance is good independently of the statistics reliability and of bind variable peeking.

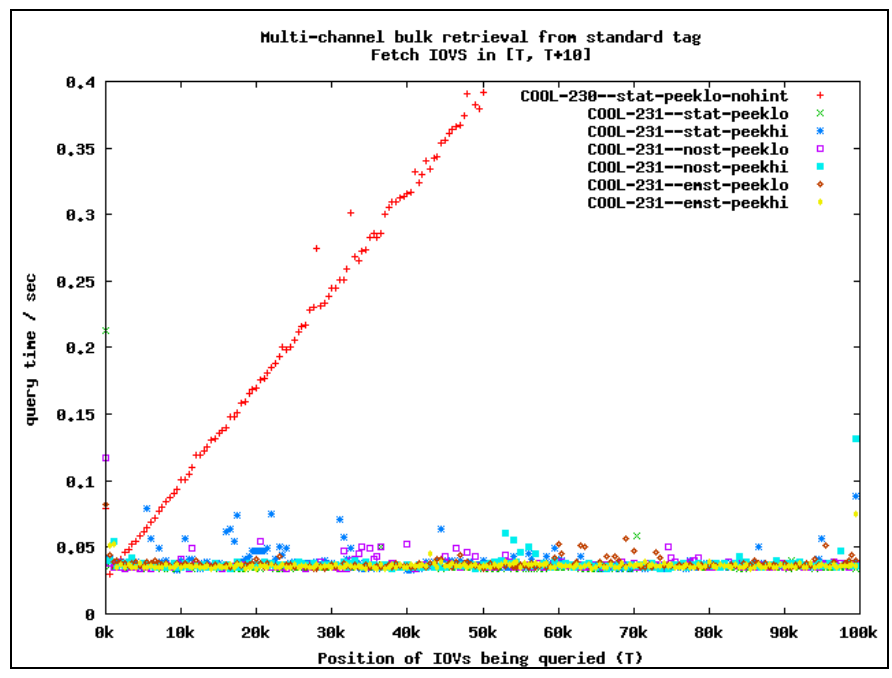

Fig. 5 - Query response times (seconds) for the retrieval of all IOVs in the time range $[\mathrm{T}, \mathrm{T}+10]$, as a function of $\mathrm{T}$ (arbitrary units), in the specific use case of "multi-version retrieval from a tag". One curve is given for release COOL 2.3.0 and the other six for release 2.3.1, under several scenarios of "statistics gathering" and of "bind variable peeking", as explained in the text. Oracle Optimizer "hints" are enabled (this is the default) for the curves plotted for 2.3.1.

\section{2) Scalability tests}

Scalability tests for data insertion and retrieval using very large samples of simulated data have been another key area of activity for the COOL project in 2008. Using the current ATLAS estimates [11] for the conditions data volumes that will be generated by the experiment when the LHC is fully operational at its design energies and beam intensities, COOL databases simulating the data samples collected by ATLAS after several years of data-taking have been produced.

Several types of conditions data are being stored by ATLAS using COOL. The bulk of the data volume is expected to come from the "detector control systems" (DCS) data recording quantities such as temperatures and voltages at the time of data taking. These are typically simple vectors of floating point numbers, which exist in a single version in COOL for each validity time as they result from a direct measurement. The total data volume from this source is expected to be around 200 GB per year, including COOL metadata and data payload as well as the associated Oracle indexes. A second important category of data stored by ATLAS using COOL 
includes computed alignment and calibration corrections for the various sub-detector components: the quantities associated to these data items have a much more complex internal substructure and may exist in several versions corresponding to different computation algorithms, but their total data volume per year is still significantly lower than that coming from DCS.

For the purpose of the COOL scalability tests described in this section, several ATLAS-like data samples were produced. To start with, one database simulating the full COOL data volume in ATLAS after one year was produced, including both DCS and calibration data for all of the ATLAS subdetectors. Other samples were produced to simulate each of the DCS and calibration data for a single sub-detector, representing equivalent data volumes ranging from one to ten years of LHC data taking for that particular data item: using a single sub-detector is enough to test scalability up to the largest expected table sizes, as the data from different subdetectors are stored in separate tables in the present ATLAS deployment model.

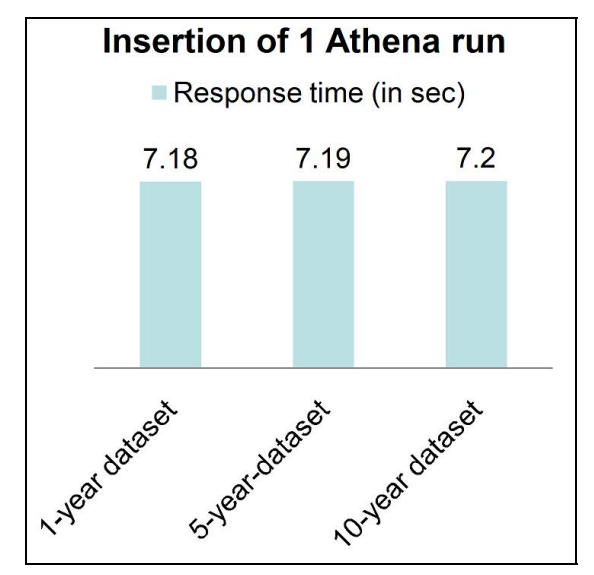

Fig. 6 - Response times (seconds) for inserting one additional data sample representing one "Athena run" of ATLAS DCS data (approximately one hour of LHC data taking) into COOL databases that already contain data volumes equivalent to one, five or ten years of LHC data taking.

One of the main goals of these tests was to check that the response times for data insertion into and data retrieval from these databases does not significantly increase as more and more data are stored. All such tests were successful. In the case of the data samples representing one particular subset of the ATLAS DCS data, for instance, it was found that the response time for inserting a data sample roughly equivalent to one hour of LHC data-taking (one "Athena run") into COOL databases already containing the equivalent of one, five or ten years only increased from 7.18 to 7.20 seconds, as shown in Fig. 6. Using the same data samples, it was found that the response time for retrieving the most recent Athena run (i.e. the one at the end of all relevant tables) from these databases only increased from 3.5 to 3.8 seconds (when data need to be retrieved from disk, while the response time is constant at approximately 0.34 seconds if the data are already in the Oracle server cache).
From the point of view of performance, the tests described above indicate that no major showstoppers are expected in data insertion into or retrieval from the extremely large COOL database tables resulting from several years of LHC data taking. It should be mentioned in any case that the option of physically partitioning these tables is being considered, as this may ease the data management operations on these large data volumes. Several alternatives are being evaluated, including the Oracle partitioned table technology.

\section{3) Deployment support}

Another important activity in the area of performance optimization in 2008 consisted in providing support to the experiments, during the tests they performed using their final deployment setup for data taking. Several improvements to the COOL software have been implemented or planned to react to problems identified during these tests. These issues are often complementary to those addressed in the tests on simulated data described in the two previous subsections.

One of the problems identified by the ATLAS database administrators during the 2008 tests, for instance, was that the list of largest consumers of server resources included several queries that simply count the IOVs for a given selection, rather than fetching the actual associated rows. This was understood as being a side-effect of a feature introduced in earlier COOL releases, allowing users to be able to know the number of IOVs that a given query would return, before actually executing it. This problem had been reported previously, but it was only addressed with high priority in 2008, after fixing several other performance issues (mentioned in a previous subsection) that were consuming even larger server resources and making it relatively less important. In agreement with the users, a patch was finally added in COOL 2.6.0 to make sure that these counting queries are only executed on demand, removing the performance problem reported by the ATLAS DBAs.

Another problem identified during the ATLAS 2008 tests is that COOL presently requires separate Oracle client sessions to read data from the tables associated to different COOL "databases", i.e. different logical collections of conditions data. The deployment model adopted by ATLAS foresees that a reconstruction job must read data from several COOL databases, associated to different sub-detectors, which are hosted on the same physical Oracle server although owned by different schemas. As a consequence, each job ends up opening several simultaneous Oracle sessions to the same database server, wasting resources. It is therefore planned that a future COOL release will allow sharing the same session to read data from several COOL databases that are visible from that session. This will also require changes in the COOL transaction management model to allow users to manually start and commit transactions, as the same transaction context will be shared when accessing several COOL databases through the same session. It should also be noted that a complementary solution to this use case may come from the 
deployment of a CORAL server, as previously described in section II.A.

\section{CONCLUSIONS}

The status and outlook of COOL software development and of the deployment of COOL relational database services has been reviewed in this article. The setup of distributed relational database services for COOL is by now complete for both ATLAS and LHCb and is being extensively tested. COOL software development has also been steadily focusing on deployment-oriented issues, performance improvements above all. In summary, the COOL project is now ready for supporting LHC data-taking.

\section{ACKNOWLEDGMENT}

We are grateful to the COOL user community for their continuous feedback about the software and suggestions for its improvement. We also thank the SPI, CORAL, FroNTier and ROOT teams for their help in improving the COOL code and development infrastructure.

The Oracle performance tests and optimizations described in this paper benefitted enormously from the help and consultancy offered by the IT-DM Physics Database Service team at CERN and in particular from the suggestions of Luca Canali, as well as from the feedback from the ATLAS DBA team at CERN and the collaboration of several DBA teams at Tier1 sites. All these contributions are gratefully acknowledged.

\section{REFERENCES}

[1] LCG Conditions Database (COOL) Project home page. http://lcgapp.cern.ch/project/CondDB

[2] A. Valassi et al., "LCG Conditions Database Project Overview", proceedings of CHEP04, Interlaken (September 2004). http://indico.cern.ch/contributionDisplay. py? contribId $=447 \&$ confId $=0$

[3] A. Valassi et al., "COOL Development and Deployment", proceedings of CHEP06, Mumbai (February 2006) and references therein. http://indico.cern.ch/contributionDisplay.py? contribId $=337 \&$ confId $=048$

[4] M. Clemencic et al., "COOL Development and Deployment Status", presented at CHEP07, Victoria (September 2007), unpublished. http://indico.cern.ch/contributionDisplay.py? contribId $=204 \&$ confId $=3580$

[5] M. Clemencic et al., "COOL Performance Tests and Optimization", presented at CHEP07, Victoria (September 2007), unpublished. http://indico.cern.ch/contributionDisplay.py? contribId $=205 \&$ confId $=3580$

[6] A. Valassi, "Distributed Deployment of Relational Database Services for the LHC Computing Grid: the COOL Conditions Database Example", presented at NEC2007, Varna (September 2007), unpublished, and references therein.

http://nec2007.jinr.ru/programme.asp

[7] D. Duellmann et al., "LCG 3D Project Status and Production Plans", proceedings of CHEP06, Mumbai (February 2006) and references therein. http://indico.cern.ch/contributionDisplay.py? contribId $=333 \&$ confld $=048$

[8] M. Girone, "Distributed Database Services", WLCG Workshop, CERN (November 2008), unpublished. http://indico.cern.ch/contributionDisplay.py? contribId $=10 \&$ confId $=32660$

[9] Z. Molnar et al., "CORAL Development Status and Plans", submitted to NSS 2008, Dresden (October 2008).

[10] A. Valassi and R. Basset, "COOL Performance Optimization using Oracle Hints", CERN Database Developers Workshop (July 2008), unpublished. http://indico.cern.ch/contributionDisplay.py? contribId $=7 \&$ confId $=36288$

[11] R. Hawkings, "ATLAS Conditions Database Data Volumes and Workloads" (May 2007), unpublished. https://twiki.cern.ch/twiki/bin/view/Atlas/CoolRefWork 